\title{
Heart Rate Responses to Acoustic Stimuli in Prenatal Chick Embryos
}

\author{
Kenji Moriya $^{\mathrm{a} *}$, Yuya Chiba ${ }^{\mathrm{b}}$ and Yoshiko Maruyama ${ }^{\mathrm{c}}$ \\ ${ }^{a}$ Department of Production Systems Engineering, National Institute of Technology, Hakodate college, \\ Tokura 14-1, Hakodate city Hokkaido, 042-8501, Japan \\ ${ }^{\mathrm{b}}$ Support Center for Engineering Education, National Institute of Technology, Hakodate college, \\ Tokura 14-1, Hakodate city Hokkaido, 042-8501, Japan \\ ${ }^{c}$ Department of Production Systems Engineering, National Institute of Technology, Hakodate college, \\ Tokura 14-1, Hakodate city Hokkaido, 042-8501, Japan \\ *Corresponding Author: moriya @hakodate-ct.ac.jp
}

\begin{abstract}
Changes of heart rate fluctuations (HRFs), which are HR variabilities and HR irregularities with various periods (e.g., in several beats, in a minute, 20-60 min (i.e. ultradian rhythms), and daily rhythms (i.e. circadian rhythms)), and autonomic nervous system activities in the prenatal chick embryos exposed to acoustic stimuli (classical and rock music, and hatchlings cheeps) were investigated. As a result, although frequency of HRFs in the rock music period were higher compared with the classical music, distinctive changes of autonomic nervous system activities were not observed in both music. In addition, characteristic HR responses and autonomic nervous activities in the cheep sound period also did not occur. A direct influence of acoustic stimuli might appear after hatching, or in prenatal embryo, vibrations with other eggs caused by the hatching activity might affect physiological functions compared with acoustic stimuli. Further experiments under other stimuli (e.g., vibration, maternal heart beat sound) are required.
\end{abstract}

Keywords: acoustic stimulus, hatchlings cheeps, prenatal chick embryo, heart rate fluctuation.

\section{Introduction}

\subsection{Chick embryos}

Chick embryos develop externally by supplying suitable temperature and oxygen, which provides multiple advantages for studying fetal development, such as the ability to measure embryonic physiological parameters that are not influenced by maternal health conditions and to assess responses to changes in incubation environment (e.g., temperature, humidity, and oxygen concentration) $)^{(\mathrm{e} . \mathrm{g}, \mathrm{1}, \mathrm{-3)}}$. Although the respiration of chick embryos changes from gas exchange through the egg shell to breathing with lungs during hatching, the measurement of cardiovascular parameters is possible even in periods of drastic respirational change, i.e., the prenatal period. Therefore, physiological parameters in chick embryos have been investigated to be a suitable and independent embryonic measurement model, particularly for cardiovascular systems, during the prenatal and hatching periods ${ }^{\text {(e.g., }}{ }^{-48)}$.

\subsection{Influence of music on embryos}

Prenatal exposure to music and sounds with $1 / \mathrm{f}$ fluctuation has been reported to be beneficial to a fetus. However, in mammals, even when music with 1/f fluctuation is played, the body of the mother acts as a filter, and it is unclear which frequency components reach the fetus. In addition, the fetal response to music is affected by the physiology of the mother. In avian embryos, however, there is no such maternal influence, and embryo responses to music can be easily determined. We have shown that eggshells possess features analogous to low-pass filters, which suggests that like the mother's body, eggshells transmit sound frequencies with $1 / \mathrm{f}$ fluctuation.

Pearson et al. have shown, based on heart rate (HR) measurement experiments on avian embryos, that exposure to hatchling cheep sounds activates the hatching activity of embryos ${ }^{(9)}$. Thus, hatchling cheeps likely constitute an important and recognizable frequency for birds.

In this study, we monitored the HR fluctuations (HRFs) and autonomic nervous system activity in chick embryos in 
response to sounds of bird cheeps and music. Two different types of music were used: classical music, which is considered to have a $1 / \mathrm{f}$ fluctuation component, and rock music, which has a fast tempo with many high frequency components.

\section{Materials and Methods}

\subsection{Instantaneous HR (IHR) measurements of chick embryos}

Fertile eggs of broiler chickens were purchased from a local hatchery. Incubation of chick embryos started on Day-0 (Espec SH-221, forced draft chamber), and generally chickens hatch on Day-21. Chicken eggs were incubated at $38^{\circ} \mathrm{C} \pm 2{ }^{\circ} \mathrm{C}$ temperature and $50 \%-60 \%$ relative humidity and turned automatically every $3 \mathrm{~h}$. This condition was also maintained during experiments of exposure to sounds. Electrocardiogram readings (ECG) were taken every $24 \mathrm{~h}$ starting from Day-16 of incubation, which marks the beginning of autonomic nervous system (i.e., sympathetic and parasympathetic nervous systems) development in embryos, before hatching on Day-20. IHR was determined offline based on adjacent $\mathrm{R}$ waves on the $\mathrm{ECG}^{(6-8)}$.

Experiments were conducted at the same time each day. The type and duration of sound administered is summarized in Figure 1A and 1B. For music experiments, measurements were obtained over approximately $2 \mathrm{~h}$, with an initial control period of $25 \mathrm{~min}$, followed by $25 \mathrm{~min}$ each of classical and rock music and 25 min of no sound after each music type. For hatchling sound experiments, measurements were obtained over $75 \mathrm{~min}$, starting with after $25 \mathrm{~min}$ of an initial control period, followed by $25 \mathrm{~min}$ of hatchling cheeps and lastly 25 mins of no sound. Eight measurements were recorded both during the music and the hatchling sound experiments. Because the number of measurements was small $(\mathrm{N}=8)$, the results were not

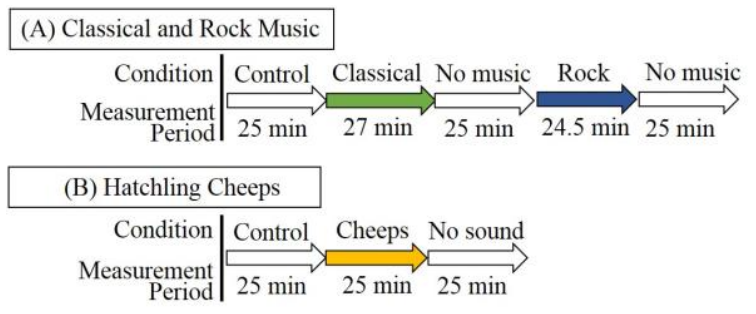

Fig. 1. Experimental protocol. (A) : two types of music. (B) : hatchlings cheeps.

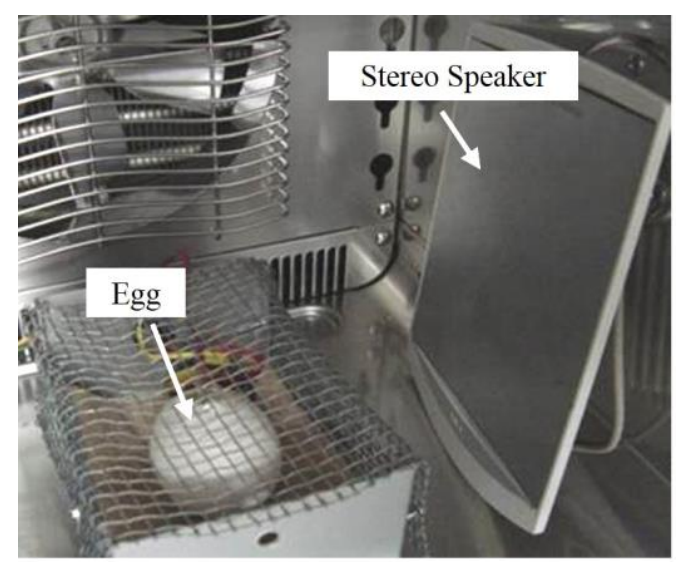

Fig. 2. Experimental environment of the exposure to the sound stimuli.

statistically analyzed.

\subsection{Sound Stimuli}

Classical and rock music were chosen for this study due to their contrasting differences. Classical music is generally used for prenatal exposure and is considered to have a soothing effect due to augmentation of the parasympathetic nervous system as it contains the $1 / f$ fluctuation. In contrast, rock music is extremely pounding and includes highly variable frequencies, which trigger the sympathetic nervous system and increase HRF. For the music experiments, the second movement of Piano Concerto No. 21 by Mozart (6 min $47 \mathrm{sec}$, repeated four times) was used as classical music, and "Painkiller" by Judas Priest (6 min $6 \mathrm{sec}$, repeated four times) was used for the rock music. This music was selected at our discretion by reasons as follows: time of this song was approximately same as that of the classical music: its beat/tempo was fast and pounding.

The egg which was put in the metal mesh box to reduce the $50 \mathrm{~Hz}$ hum noise mixed in ECG signal was placed in the center at distance of $5 \mathrm{~cm}$ each from the commercial speakers (Authentic Inc., NXT flat panel speaker, size $\mathrm{W} 140 \times \mathrm{H} 123 \mathrm{~mm}$, maximum output $3 \mathrm{~W}+3 \mathrm{~W}$, the sub-woofer speaker was not used) (Fig. 2). The inner size of incubator is $\mathrm{W} 300 \times \mathrm{H} 300 \times \mathrm{D} 250 \mathrm{~mm}$, and temperature and humidity were kept at the usual incubation condition. The maximum volume of classical and rock music was set to the same level, and it was kept in all experiments. The hatchling cheeps were also set to a constant volume and it was used in all experiments.

2.3 Spectrum analysis of IHR and indices of 


\section{autonomic nervous system activity}

Since IHR is not at regular time-intervals, IHR data was resampled at $10 \mathrm{~Hz}$ by linear interpolation ${ }^{(10)}$. Then, power spectrum analysis of IHR was performed using fast Fourier transform after Hamming window was applied ${ }^{(11)}$. To reduce the noise effect (incorrect IHR), each 5-min interval was divided into 512 points (i.e. $52.1 \mathrm{sec}$ of segment) and spectrum components of each segment were averaged. Thus, the frequency resolution is approximately $0.02 \mathrm{~Hz}$ and maximum frequency depends on a mean HR (e.g. if mean $\mathrm{HR}$ is $360 \mathrm{bpm}$, that is equivalent to $6 \mathrm{~Hz}$, meaningful maximum frequency is $3 \mathrm{~Hz}$ according to the sampling theorem $)^{(10)}$.

In chick embryos, low frequency (LF) and high frequency (HF) components are $0.04-0.15 \mathrm{~Hz}$ and $0.1-1.0$ $\mathrm{Hz}$, respectively ${ }^{(8,10,12)}$. HF component is the index of parasympathetic nervous system activity and the LF/HF ratio is the index of sympathetic nervous system activity. Normalized HF components (\%HF), represents the ratio of $\mathrm{HF}$ component to $\mathrm{HF}+\mathrm{LF}$ and is used as an index of parasympathetic nervous system activity and reduces influence from very low frequency component. Each component was calculated by a numerical integration ${ }^{(10,13)}$. In General, in chick embryo, parasympathetic nervous function develops from around Day-14, and sympathetic nervous function develops from around Day-16 ${ }^{(6)}$.

\section{Results}

\subsection{Spectra of music and hatchlings cheep in air and within the egg}

Figure $3 \mathrm{~A}$ and $3 \mathrm{~B}$ show both spectra of the classical and the rock music (i.e., frequency components), respectively. The horizontal axis indicates frequency $(\mathrm{kHz})$ and vertical axis indicates normalized spectrum power. Black solid lines are spectra of music that was recorded in the air (music), red solid lines are those within the egg (in egg shell). The in situ egg spectra (red lines) were estimated from frequency characteristics of eggshells determined in our previous study ${ }^{(14)}$. It should be noted that in our previous work, the spectra measurements were recorded from eggshells only because the egg yolks and whites had been removed; thus, the shape of the spectrum may be different for egg yolks. However, in the current study, chicken embryos at Day 16 almost completely filled the interior of the egg, and the filtering of sound by the amniotic fluid was considered negligible.

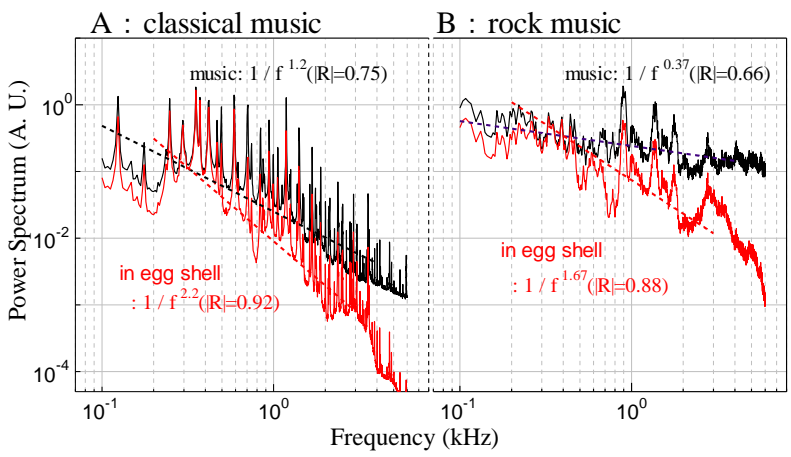

Fig. 3 Spectra of music in air (music, black solid lines) and within the egg (in egg shell, red solid lines): A. classical music, B. rock music, respectively. The $\alpha$ of $1 / \mathrm{f}^{\alpha}$ indicates the gradient of the spectrum (i.e., spectrum structure) determined from least square method, and dotted line is an approximated equation $P(f)=1 / f^{\alpha}$ and $|R|$ is its correlation coefficient.

The $\alpha$ is the gradient of power spectrum on the log-log plot; therefore, $\alpha=1$ means the spectrum has a $1 / \mathrm{f}$ structure or a $1 /$ f fluctuation. The gradient $\alpha$ is determined by least square method (i.e., linear approximation) with steepest descent. The gradient of each spectrum is also shown in Figure 3. Because the music changes in volume and melody from beginning to end, the spectra shown are of the selected arbitrary $5 \mathrm{~s}$ segments. The spectrum of a music piece can certainly change over time; however, we confirmed that on an average, a characteristic spectrum can be obtained.

The spectrum of classical music in the air was close to $1 / \mathrm{f}\left(\alpha=1.2,1 / \mathrm{f}^{1.2}\right)$, which indicates the high frequency components were low. In contrast, the spectrum gradient of rock music was $\alpha=0.37,1 / \mathrm{f}^{0.37}$, which indicates this $5 \mathrm{~s}$ of rock music contains many high frequency components. The spectra (especially, higher component than $3 \mathrm{kHz}$ ) of music heard by the embryo inside the egg shell were attenuated by low-pass filter effect of the egg shell. However, even above $3 \mathrm{kHz}$, the rock music spectrum, which includes many high frequency components, was more powerful and larger overall than the classical music spectrum. The gradients of the spectra in the egg shell (i.e., after low-pass filtered) were $1 / \mathrm{f}^{2.2}$ (classical music) and $1 / \mathrm{f}^{1.7}$ (rock music) respectively.

The spectra of hatchling cheep in air (cheep) and within the egg (in egg shell) are shown in Figure 4. The frequency of chicken hatchling cheeps was around $3.5 \mathrm{kHz}$. Low frequency band (lower than approximately $600 \mathrm{~Hz}$ ) 
was attributed to sounds of hatchlings walking or ambient noise.

The hatchling cheeps were played at a slightly higher volume than would be normally heard.

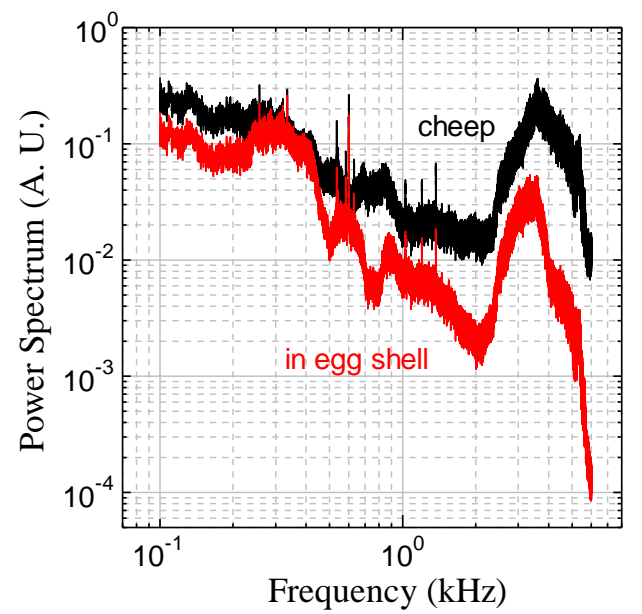

Fig. 4 Spectra of a chicken hatchling cheep in air (cheep, black solid line) and within the egg (in egg shell, red solid line). The frequency component of chicken hatchling cheep was around $3.5 \mathrm{kHz}$.

\subsection{Effect of music on HRF and autonomic nervous system activity}

The experiments were conducted in eight embryos. IHR fluctuation and autonomic nervous system activity indices at 5-min intervals for an individual embryo at Day-17 and Day-18 of incubation are shown in Figures 5 and 6 , respectively. The Y-axis in the upper panel represents IHR. In the bottom panel, the primary Y-axis (left) indicates the ratio of low frequency (LF) to high frequency (HF) components (LF/HF ratio) that was used as an index for sympathetic nervous system activity, whereas the secondary Y-axis (right) indicates the percentage of HF components as an index of the parasympathetic nervous system activity. The horizontal axis represents time in min.

Compared with the baseline HR, significant changes were not observed in a Day-17 embryo in response to classical music (Fig. 5). In contrast, an approximately first 7-min exposure to rock music appeared to induce large HR accelerations (or tachycardia, which is transient HR acceleration) with magnitude of approximately $100 \mathrm{bpm}$ repeatedly. Subsequently, brief HR decelerations (or bradycardia, which is transient HR deceleration) with magnitude of approximately $50 \mathrm{bpm}$ occurred for $5 \mathrm{~min}$ accompanying with baseline HR decrease of about $20 \mathrm{bpm}$ until the baseline HR recovered to the baseline level prior to the bradycardia. In the last $10 \mathrm{~min}$ of rock music, distinctive HRF was not observed. The parasympathetic nervous system activity increased while the embryo was

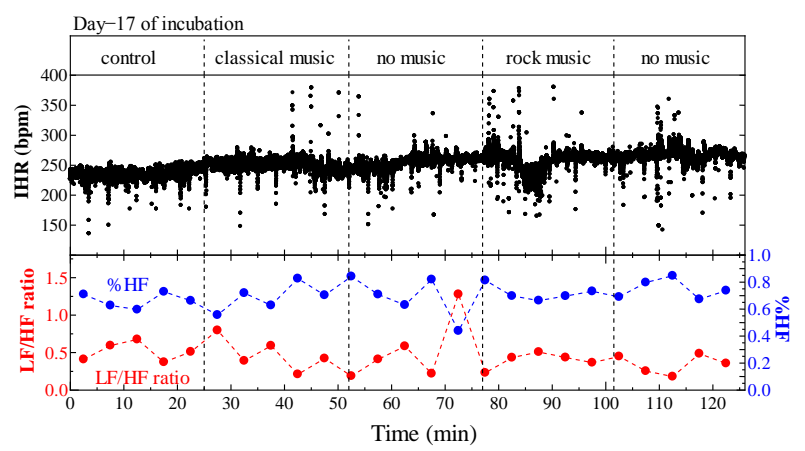

Fig. 5 Measurements of instantaneous heart rate fluctuation (upper panel) and autonomic nervous system activity indices at 5-min intervals (bottom panel) in a Day-17 embryo exposed to music. Black dots are IHRs, and red and blue closed-circles show the index of sympathetic (left Y-axis) and parasympathetic (right Y-axis) nervous system activity, respectively.

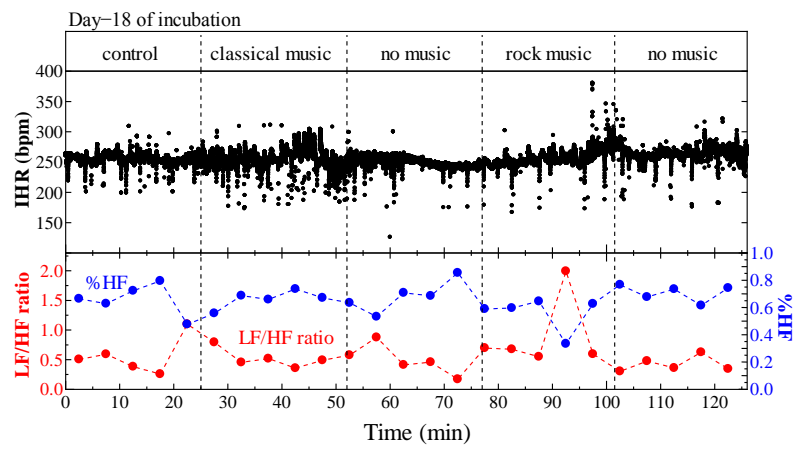

Fig. 6 Measurements of instantaneous heart rate fluctuation and autonomic nervous system activity indices at 5-min intervals in a Day-18 embryo exposed to music. This embryo was the same individual as the embryo shown in Fig.5.

exposed to the classical music. However, when rock music was played, change in parasympathetic nervous system activity was minimal.

The occurrences of both HR acceleration and HR deceleration increased in a Day-18 embryo when classical music was played (Fig. 6). At approximately $47 \mathrm{~min}$, baseline HR decreased to approximately $20 \mathrm{bpm}$ and then recovered about 2 min later to the baseline level prior to the bradycardia. Although both tachycardia and bradycardia 
occurred, nervous system activity indices suggested that the parasympathetic nervous system seemed to be dominant. During the no music period after classical music, HR decelerations still occurred, but HR accelerations were not observed. However, when rock music was played next, HR accelerations with magnitude of approximately $20 \mathrm{bpm}$ were frequently induced again and baseline HR increased towards to the end of rock music period. After heard the rock music, baseline HR decreased to that of before rock music periods.

In other individuals, although frequency of HRF occurrence tended to be higher when exposed to rock music, a characteristic HR fluctuation pattern or autonomic nervous system activity distinctive in the type of music could not be elucidated. In Day-20, because baseline HR changes, large HR accelerations, and HR decelerations often occurred generally, to distinguish whether HRF in music period was usual HRF or music effects was extremely difficult.

\subsection{Effect of hatchling cheeps on HRF and autonomic nervous system activity}

Measurements of IHR and autonomic nervous system activity indices at 5-min intervals for an individual embryo, which was different individual from embryo shown in Fig. 5 and 6, at Day-18 and Day-19 are shown in Figures 7 and

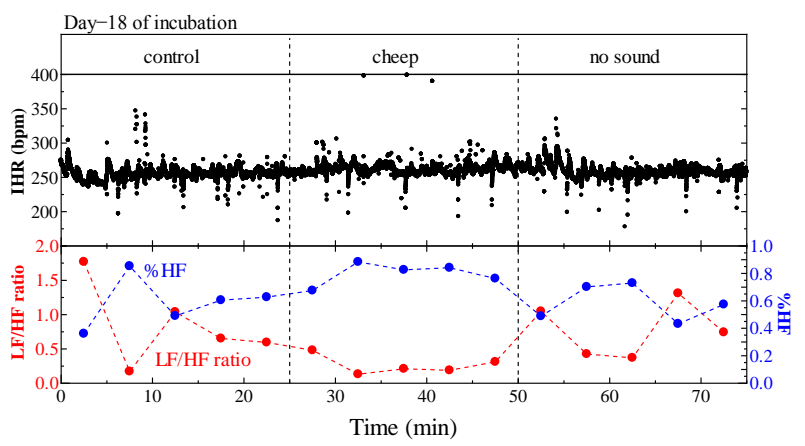

Fig. 7 Instantaneous heart rate and autonomic nervous system activity indices in a Day-18 embryo exposed to hatchling cheeps.

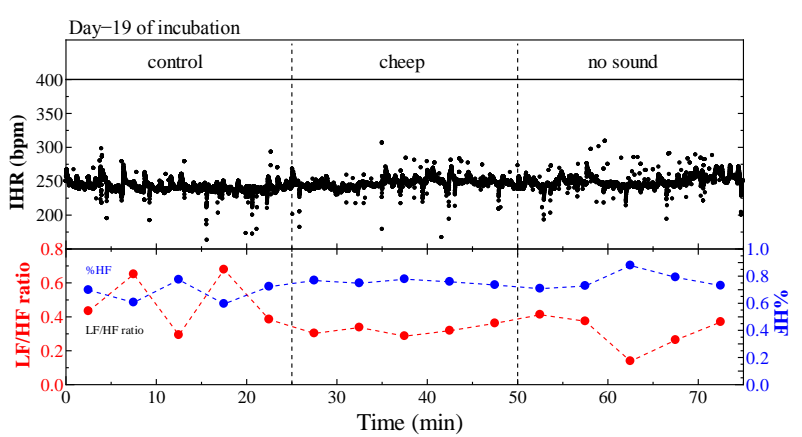

Fig. 8 Instantaneous heart rate fluctuation and 5-min measurements of autonomic nervous system activity indices in a Day-19 embryo exposed to hatchling cheeps
8 , respectively. This embryo is the same individual embryo shown in Figs. 5 and 6. The graph axes are the same as for Figures 5 and 6 . When the embryo was exposed to hatchling cheeps, large baseline HR changes did not appear and frequency and magnitude of HR accelerations and HR decelerations seemed to be similar to those of control period (Fig.7). However, although distinctive HRF, especially HR decelerations, was not observed during cheep-period, parasympathetic nervous system activity increased or was dominant. The Day-19 embryo also did not exhibit characteristic HRF and showed minimal changes in the sympathetic and parasympathetic nervous system activity indices (Fig. 8). A characteristic HR fluctuation dependent on the exposure to hatchling cheeps was not noted in other embryos and the other incubation days. In addition, the autonomic nervous system activity changed in various patterns. In addition, although the autonomic nervous system activity changed in various patterns which were observed in controlled incubation, a characteristic pattern in response to hearing hatchling cheeps did not appear.

\section{Discussion}

\subsection{HR response to sound stimulation}

Prenatal chick embryos showed a more striking response to rock music, as evident from the increase in HR, compared with classical music. However, substantial effects of rock music on autonomic nervous system activity were not detected. It may be that music stimulation does not greatly influence physiological parameters, particularly autonomic nervous system activity, or that the effect is insensibly until the autonomic nerve function is completely developed, just prior to hatching. In our experiments, classical and rock music were alternately played only for short intervals of $25 \mathrm{~min}$ per day each. Different patterns of HRF and autonomic nervous system activity, particularly parasympathetic nervous system, might be observed when embryo is exposed to classical music for more long term. However, as a key point, because the high frequency component (i.e., more than approximately $3 \mathrm{kHz}$ ) inside of the egg shell is attenuated, music which has a $1 / \mathrm{f}$ fluctuation structure could affect on chicken after hatching. In mammalian fetus, a maternal abdomen (i.e., an amnionic fluid) also has a low-pass filter effect. Therefore, 
comfortable music influences maternal condition, in consequence, it might affect a fetus indirectly.

Pearson et al. reported on the phenomenon that hatching of one embryo activated the hatching of others. We had hypothesized that to hear the cheeps of other hatched chick(s) promotes augmentation of the sympathetic nervous system activity that is required for the hatching. However, distinctive changes of HRF and autonomic nervous system, particularly sympathetic nervous system, activity were not observed also in embryos exposed to hatchling cheeps even at Day 19 that embryo already started hatching process. This may be due to vibrations and/or collisions with other eggs caused by the hatching activity or the movements of the newly emerged hatchlings. In Day-20, which is 1 day before the emergence from the egg shell, to distinguish whether HRF in music period was usual HRF or music effects was extremely difficult. This result indicates even if autonomic nervous system responded to acoustic stimuli, it is an inconsiderable effect compared with the hatching event.

\subsection{Future investigations}

As other possibility, the presence of $1 /$ f fluctuation in the maternal heartbeat rhythm is indicative of why prenatal exposure to music with $1 / \mathrm{f}$ fluctuation is beneficial for the embryos. Therefore, to investigate the response to vibrations and/or sound stimuli of HR of parent birds that are incubating the embryos is necessary for the next research.

\section{Acknowledgment}

A part of this study was supported by a Grant-in-Aid for Scientific Research (K.M. JP23700547, JP16K06397) from the Japan Society for the Promotion of Science.

\section{Additional statement}

This research was approved by the Life Ethics Committee of NIT, Hakodate College.

\section{References}

(1) Hiroshi Tazawa, Amos Ar, Gefen, E., Kenji Moriya, and James T. Pearson: "Effects of incubator humidity on embryonic heart rate in the ostrich", Proc. 10th European Poultry Conference. pp.843-847, 1998.

(2) Hiroshi Tazawa, Kenji Moriya, Akihiko Tamura, Takashi Komoro, and Ryuichi Akiyama: "Ontogenetic study of thermoregulation in birds", Journal of Thermal Biology. 26:281-286, 2001.

(3) Akihiko Tamura, Ryuichi Akiyama, Kenji Moriya, Edward M. Dzialowski, Warren W. Burggren, and Hiroshi Tazawa: "Heart rate responses to cooling in emu hatchlings", Comparative Biochemistry and Physiology 134/4: 829-838.2003.

(4) Hiroshi Tazawa, Hikaru Mitubayashi, Makoto Hirata, Joachim Höchel, and James T. Pearson: "Cardiac rhythms in chick embryos during hatching", Comparative Biochemistry and Physiology, 124A: 511-521, 1991.

(5) Ryuichi Akiyama, Akira Matsuhisa, James T. Pearson, and Hiroshi Tazawa: "Long-term measurement of heart rate in chicken eggs", Comparative Biochemistry and Physiology, 124A: 483-490, 1999.

(6) Kenji Moriya, Joachim Höchel, James T. Pearson, and Tazawa, H: "Cardiac rhythms in developing. chicks. Comparative Biochemistry and Physiology. 124, 463-470, 1999.

(7) Kenji Moriya, K., James T. Pearson, Warren W. Burggren, Armos AR, and Tazawa, H: "Continuous measurement of instantaneous heart rate and It's fluctuations before and after hatching in chickens", Journal of Experimental Biology, 203, 895-903, 2000.

(8) Kenji Moriya, Ryuichi Akiyama, Edward M. Dzialowski, Warren W. Burggren, and Hiroshi Tazawa: "Development of heart rate circadian rhythm in chickens", Avian and Poultry Biology Reviews, Vol.15, No.3/4, pp.211-218, 2004.

(9) James T. Pearson, Kenji Moriya, Yanone, M., and Hiroshi Tazawa: "Development and regulation of heart rate in embryos and hatchlings of gulls (Larus schistisagus and Larus crassirostris) in relation to growth", J ournal of Comparative Physiology, B, 170: 429-438, 2000.

(10) Kenji Moriya, Yasuhiro Chiba, Hitoshi Yoneta, Ryuichi Akiyama, and Hiroshi Tazawa: "Simultaneous measurement of instantaneous heart rate and breathing activity in newly hatched chicks", Br. Poult. Sci. Vol.44, No.5, pp.761-766. 2003.

(11) Harris, F.J.: "On the use of windows for harmonic analysis with the discrete Fourier transform", Proceedings of the IEEE, 66: 51—83., 1978. 
(12) Sorange Akselrod, David Gordon, F. Andrew Ubel, Daniel C. Shannon, A. Clifford Barger, and Richard J. Cohen: "Power spectrum analysis of heart rate fluctuation: a quantitative probe of beat-to-beat cardiovascular control", Science 213, 220-222, 1981.

(13) Kenji Moriya, Shiita Ishigaki, Nobuo Ezaki, Ikusaburou Kurimoto, and Masahiro Nakagawa: "Influence on brain activity during and after a study break using different types of music", Proceedings of The 5th IIAE International Conference on Intelligent Systems and Image Processing 2017, pp.152-156, 2017.

(14) Yuki Nakajima and Kenji Moriya: "Heart rate responses to sound stimulus with various frequencies", Research reports of National Institute of Technology, Hakodate College, Vol.45, pp.35-42, 2011. 